\title{
FUSION OF COLOR AND MODIFIED WEBER'S LAW DESCRIPTOR BASED HUMAN SKIN DETECTION
}

\author{
${ }^{1}$ Prema, C. and ${ }^{2} \mathrm{D}$. Manimegalai \\ ${ }^{1}$ Department of CSE, Jayaraj Annapackiam CSI College of Engineering, Nazareth, TN, India \\ ${ }^{2}$ Department of IT, National Engineering College, Kovilpatti, TN, India
}

Received 2014-01-04; Revised 2014-01-13; Accepted 2014-04-19

\begin{abstract}
Skin detection in images or videos plays a vital role in a wide range of image processing applications like face detection, face tracking, gesture analysis, human-computer interaction etc. Due to its high processing speed and invariant against rotation, it has received significant interest in pattern recognition and computer vision. The robustness of skin detection using color information depends on real world conditions such as background, noise, change of intensity and lightening effects. This situation can be improved by using texture as a descriptor to extract skin pixels in images. This study proposes color based skin detection algorithm (fusion of Cheddad's approach with $\mathrm{C}_{\mathrm{r}}$ of $\mathrm{YC}_{\mathrm{b}} \mathrm{C}_{\mathrm{r}}$ color space) with a texture based skin location algorithm called Modified Weber's Law Descriptions (MWLD) to evaluate region features. MWLD is based on the fact that the Human Visual System (HVS) is more sensitive in lumens contrast than absolute luminance values. In MWLD, the differential excitation and gradient orientation of the current pixel are considered to extract the texture features. For differential excitation, Just Noticeable Distortion (JND) is also used. Due to the absence of red component in Cheddad's new color space for skin detection, the detection rate is not good for real time applications. To improve this situation, the $\mathrm{Cr}$ component of $\mathrm{YC}_{b} \mathrm{C}_{\mathrm{r}}$ color space is included to obtain the color features. According to experimental results, the proposed method exhibits satisfactory performance in terms of True Positive Rate (TPR), False Positive Rate (FPR) and Accuracy with wide variations in size, color and orientation. Different window sizes for differential excitation such as $3 \times 3,5 \times 5$ and $7 \times 7$ are also taken to check the performance.
\end{abstract}

Keywords: Skin Tone Detection, $\mathrm{YC}_{\mathrm{b}} \mathrm{C}_{\mathrm{r}}$ Color Space, Modified Weber's Law Descriptor, Just Noticeable Distortion, True Positive Rate, False Positive Rate

\section{INTRODUCTION}

Automatic skin detection has become a very challenging topic during the last several decades. However robust skin detection under varying illumination condition is still challenging. An important requirement for automatic skin detection is the correct classification rate and response time. In skin detection, if skin is used as a feature then the researcher faces two major problems. First, what color space to choose and second how exactly skin color distribution should be modeled. The detailed survey for the above two problems are taken by Vezhnevets et al. (2003). In his survey he concluded that excluding color luminance from the classification process cannot help to achieve better result and how good the color space suited for skin modeling. To include luminance, Cheddad et al. (2009) used a new color space which contains the error signal derived from differentiating the grayscale map and the non-encoded-red gray scale version. In his work he discarded the red color in calculating the component Equation 1:

$$
\mathrm{I}(\mathrm{x})=\max _{\mathrm{x} \in(1, \ldots, \mathrm{n})} \mathrm{Gx}, \mathrm{B}(\mathrm{x})
$$

Experimental results were carried out using his algorithm but it is not in reasonable agreement with this assertion. Color transformations are very important in Corresponding Author: Prema, C., Department of CSE, Jayaraj Annapackiam CSI College of Engineering, Nazareth, TN, India 
computer vision. Different color spaces exist but the RGB color describes the world view in three color matrices. The luminance is present in this space and various transforms are meant to extract it. $\mathrm{The} \mathrm{YC}_{\mathrm{b}} \mathrm{C}_{\mathrm{r}}$ is widely used in digital video. In this format, luminance is represented by a single component $\mathrm{Y}$ and color information is stored as two color difference components $\mathrm{C}_{\mathrm{b}}$ and $\mathrm{C}_{\mathrm{r}}$. Components $\mathrm{C}_{\mathrm{b}}$ is the difference between the blue component and the reference value and component $\mathrm{Cr}$ is the difference between the red component and the reference value. The transformation used to convert from $\mathrm{RGB}$ to $\mathrm{YC}_{\mathrm{b}} \mathrm{C}_{\mathrm{r}}$ is Equatino 2:

$$
\begin{aligned}
& \mathrm{Y}=0.299 \mathrm{R}+0.587 \mathrm{G}+0.114 \mathrm{~B} \\
& \mathrm{C}_{\mathrm{b}}=0.56-\mathrm{B}-\mathrm{Y} \\
& \mathrm{C}_{\mathrm{r}}=0.71(\mathrm{R}-\mathrm{Y})
\end{aligned}
$$

Hsu et al. (2002) used $\mathrm{C}_{\mathrm{b}} \mathrm{C}_{\mathrm{r}}$ color space for face detection. They claimed that their algorithm has detected more skin-tone facial pixels. Shin et al. (2002) showed that the use of color transformation does not improve the detection rate compared to several other color transformations and also dropping of illumination component significantly worsen the separability. However skin color detection presents several important problems under uncontrolled conditions. It is affected by the illumination, it can be confused with the color of metal (or) wood surfaces and also skin color can change from person to person.

Prema and Manimegalai (2012) presented a method based on hybrid approach to detect skin detection. In their approach, the Cheddad's approach is combined with $\mathrm{Cr}$ of YCbCr color space. In Cheddad's approach, the red channel is discarded. Only green and blue channels are used to find the skin tones. Due to the absence of red channel, Cheddad's approach is not working properly in images which have poor illumination. To improve the performance, they proposed a hybrid which includes $\mathrm{Cr}$ component of $\mathrm{YCbCr}$ color space.

Texture is another important feature for skin detection which differs from color. It takes the account of the positional relation among pixels in a region of the image. It is characterized by the spatial distribution of gray levels in a neighborhood. There are different types of texture feature extraction such as statistical, geometrical, model based and signal processing methods. In recent years, more and more attention is paid to local texture features in face detection due to its more robustness to variations of facial pose, expressions, occlusion. In particular, Gabor wavelets (Liu and Wechsler, 2002) and Local Binary Patterns (LBP) which is first presented by Ojala et al. (2002) are mainly used in texture detection. LBP was successfully introduced into face recognition by Ahonen et al. (2006) and has gained much attention by researchers in face recognition. According to Caulsi and Jernigam (2002), texture features generated by Gabor filters produced preferred results. In Ahonen's face recognition method (Ahonen et al., 2006), LBP feature distributions from several regions are extracted and concatenated to produce a face descriptor.

Another important local texture descriptor is Weber's Law Descriptor (WLD) which was proposed as a simple and powerful image descriptor by Chen et al. (2010). WLD was used in face recognition by Gong et al. (2011). In WLD, the gradient orientation describes the direction of edges. In this study, Modified WLD (MWLD) is used to extract the texture features. MWLD is derived from the psychological postulates developed by Thrust one (Early Alternative) which is in the form of Equation 3:

$\mathrm{S}=\mathrm{k} \log (\mathrm{x})+\mathrm{c}$

where, $\mathrm{x}$ is the differential execution of Weber's law which is calculated by using the expression Equation 4:

$$
\operatorname{arctamp} \underset{i=1}{p-1} \frac{\mathrm{x}_{\mathrm{i}}-\mathrm{x}_{\mathrm{c}}}{\mathrm{x}_{\mathrm{c}}}
$$

And $\mathrm{c}$ is maximum weighted gradient using different gradient operators. Wang et al. (2011) used WLD to normalized illumination which is applied to recognize the face images. He created the Weber-face which performs better than the existing recognition methods.

To analyze the color image in two ways, methods that process color and texture information separately and that consider the color and texture as a joint phenomenon. Based on the results of Tope Maenpaa and Pietikäinen (2004), they suggested that the color and the texture are separate phenomenon that can (or) even should be treated individually. So in this study, the color and texture features are treated separately and fused together to detect the skin pixels. Main key problems in skin detection are detection rate and time consumption. The proposed method is simple and feasible. Experimental results show that this method has good detection rate. 
Taqa and Jalab (2010) proposed a novel Fuzzy Inference System (FIS) for skin detection, which combines both color and texture features. To increase the reliability of the skin detection process, neighborhood pixel information is incorporated into the proposed method. The color feature is represented using RGB color model, while the texture feature is estimated using three statistical measures: Standard deviation, entropy and range. The subtractive clustering-based fuzzy system method and the Sugeno type reasoning mechanism are used for modeling FISbased skin detection. The proposed approach builds a fuzzy model of skin detection from existing images within skin and non-skin regions (output data) and from both color and texture features of the skin regions (input data). The problem of fuzzy inference methos is that test set is needed to train the fuzzy model. This draw back is eliminated by thisproposed method.

Recent review presented by Mustafa and Zhu (2013) said that most of the works are based on skin color detection, which are not suitable for finding obscenity. The reason for this is that, there are many skins like objects such as beach photos, human skin like animal's fur, skin colored painting that enables false positive and negative rate. They concluded that choice of proper color model, skin segmentation algorithms and classifiers are the factors to consider in skin detection method. So, in this proposed method more importance is given to color model and skin segmentation algorithms. In stead taking a single color medel, combined approach is used tn this proposed method. To increase the skin segmentation rate texture features are also considered.

The rest of this study is organized as follows. Section II explains the method to detect the skin pixels using color features. In section III, the proposed local texture feature method is outlined. Section IV details the proposed fusion of color and texture approach for skin detection. Comparative analysis of the results that demonstrates the effectiveness of the proposed method is given in section V. Conclusion and future work are drawn in section VI

\section{COLOR FEATURE EXTRACTION}

Cheddad et al. (2009) recently have developed a new skin detection method and a fixed threshold classification technique. Their method is not enough to discriminate if a scene contains a skin color or something that looks similar to it. To rectify the above method, two or more color spaces are used by researchers (Hybrid approaches). Prema and Manimegalai (2012), this problem is reduced by introducing the $\mathrm{Cr}$ of $\mathrm{YC}_{\mathrm{b}} \mathrm{C}_{\mathrm{r}}$ color space. In this study, this hybrid approach is used to extract the color features. The utilized color transformation matrix is given as Equation 5:

$$
\begin{aligned}
& \mathrm{a}=0.298936021293775390,0,587945074451121360, \\
& 0,14020904551032500^{\mathrm{T}}
\end{aligned}
$$

where, $\mathrm{T}$ denotes transport operator to allow for matrix multiplication. The initial color transformation is given as Equation 6:

$\mathrm{Ix}=\varphi \mathrm{r} x, \mathrm{~g} \mathrm{x}, \mathrm{b}(\mathrm{x}) \otimes \infty$

New vector is formed by taking the largest elements from $\mathrm{G}$ or B Equation 7:

$$
\mathrm{Ix}=\max _{\mathrm{x} \in(1, \ldots, \mathrm{n})} \mathrm{Gx}, \mathrm{B}(\mathrm{x})
$$

The error signal is obtained by using the formula Equation 8:

$\mathrm{ex}=\mathrm{Ix}-\mathrm{I}(\mathrm{x})$

To get the empirical rule, the collected data could fit in a Gaussian curve using Expectation Maximization (EM) method which is an approximation of Gaussian Mixture Models (GMM). For finding the skin tones, the empirical rule used is given by Equation 9:

$\mathrm{f}_{\text {skin }} \mathrm{x}=$ lif $0.04 \leq \mathrm{ex} \leq 0.17$
0eleswhere

About $\mu$ and $\sigma$ are calculated by fitting the data to the Gaussian curve. If $\mu \leq 0.02, \mathrm{Cr}$ is taken to find the skin tones in complex background. The modified empirical rule to find the skin tones as Equation 10:

$$
\mathrm{f}_{\text {skin }} \mathrm{x}=\text { lif } \mathrm{c}_{\mathrm{r}}>\operatorname{\text {graythresh}}\left(\mathrm{c}_{\mathrm{r}}\right) \leq 0.17
$$

The result obtained by this method is shown in Fig. 1. 

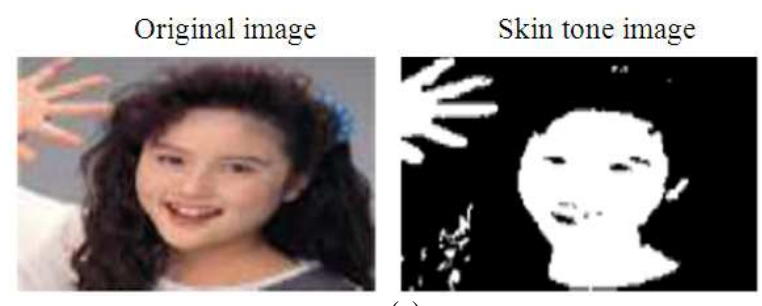

(a)

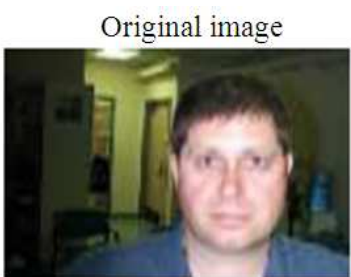

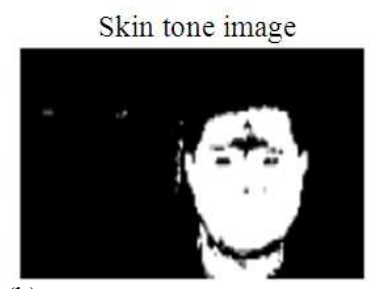

)

Fig. 1. Comparison between the original and skin tone images using (Prema and Manimegalai, 2012)

\section{MODIFIED WEBER LAW DESCRIPTOR (MWLD) FOR TEXTURE FEATURE EXTRACTION}

Based on old idea of the 'Reflectance Perception Model', an image $\mathrm{I}(\mathrm{x}, \mathrm{y})$ is regarded as the product of luminance $\mathrm{I}(\mathrm{x}, \mathrm{y})$ and the Reflectance $\mathrm{R}(\mathrm{x}, \mathrm{y})$ at each point $(\mathrm{x}, \mathrm{y})$ :

$$
\begin{array}{lll}
\mathrm{I}(\mathrm{x}, \mathrm{y})=\mathrm{L}(\mathrm{x}, \mathrm{y}) \quad \mathrm{X} & \mathrm{R}(\mathrm{x}, \mathrm{y}) \\
\mathbb{1} & \mathbb{1} & \mathbb{1} \\
\text { Image } & \text { Illu min ation } & \text { Reflection }
\end{array}
$$

According to HVS, (i) human vision is mostly sensitive to scene reflectance and mostly insensitive to illumination conditions and (ii) human vision responds to global changes in contrast rather than to global brightness levels.

\subsection{Weber's Law}

Weber's law, which is a psychological law, (AK Jain) indicates that the Human Vision System (HVS) is more sensitive to luminance contrast than absolute luminance values. If the luminance of the eye stimulus is just noticeable from the surrounding luminance, the ratio of Just Noticeable Difference (JND) is approximately constant. This is called Weber fraction. It can be expressed as Equation 11:

$$
\frac{\Delta \mathrm{I}}{\mathrm{I}}=\mathrm{K}
$$

where, $\Delta \mathrm{I}$ represents the JND and I represent the initial stimulus intensity and $\mathrm{K}$ is called Weber fraction. Weber's law is true for the perception of a variety of sensations including weight, sound intensity and light intensity. Chen et al. (2010) proposed WLD for classification of texture and face detection. WLD consists of two components: Differential excitation and orientation to capture the magnitude and direction of local intensity variations respectively.

In this study, the differential excitation component of WLD is used to capture the magnitude variations. Differential excitation gives the differences between its neighbors and current pixel as the changes of the current pixel. It is illustrated in Fig. 2.

The difference between the neighbours and the centre pixel using the filter $\mathrm{f}_{00}$ is given by the Equation 12 :

$$
\mathrm{v}^{00}=\mathrm{i}=0 \quad \Delta \mathrm{xi}=\mathrm{i}=0 \mathrm{xi}-\mathrm{xc}
$$

where, $x i(i=0,1 \ldots p-1)$ denotes the neighbors of $x i$ and $p$ is the number of neighbors. Differential excitation of the current pixel (xi) is computed as Equation 13:

$$
\arctan \frac{v^{00}}{v^{01}}=\arctan i=1 \frac{x_{i}-x_{c}}{x_{c}}
$$

Computation of the differential excitation by using the equation 13 is illustrated in Fig. 3. The two filters F00 and F01 are used to get the difference between the center and neighbouring pixels. By using differnet sizes for filters, different differential excitation are got for the same neighbouring pixels.

Fechner postulated the law of sensation which takes the general form of $\mathrm{L}=\mathrm{f}(\mathrm{I})$. He regarded that Weber fraction is obtained by differentiating the sum of JNDs. So in this study sum of JND is used to obtain the Weber fraction. Weber's law gives the justification for assuming a logarithmic mapping from input stimulus to perceived sensation. It characterizes how the $\Delta \mathrm{I}$ depends on I. It states that Equation 14:

$$
\Delta \mathrm{I}=\mathrm{k}_{\mathrm{W}} \mathrm{I}
$$




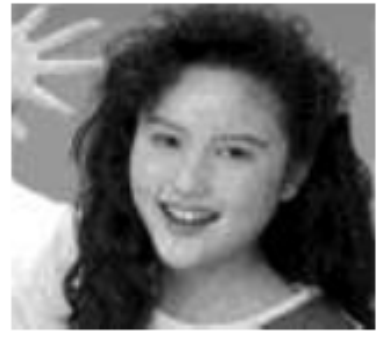

(a)

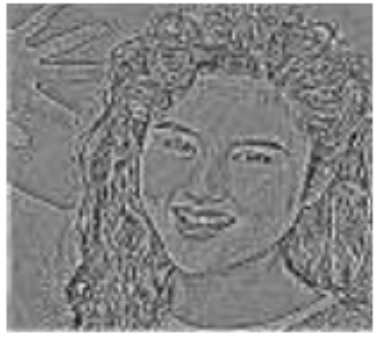

(b)

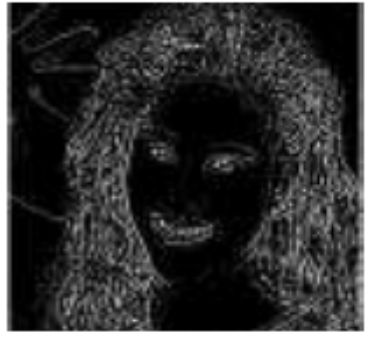

(c)

Fig. 2. (a) Grayscale image (b) output of filter (c) Weber's face

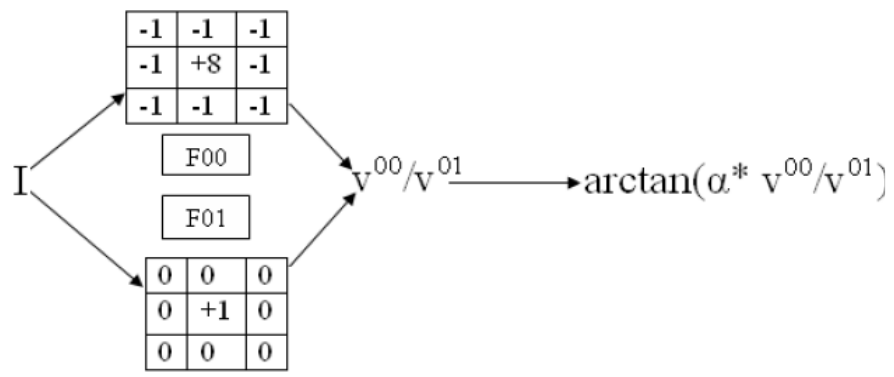

Fig. 3. Illustration of differential Excitatation calculation using filters F00 and F01

This equation is similar to the equation of straight line $\mathrm{y}$ $=\mathrm{mx}+\mathrm{c}$. This law might not hold for absolutely all values of I. For small values of I, it starts to breakdown. So the generalized formulation of Weber's law can be written as Equation 15:

$$
\Delta \mathrm{I}=\mathrm{k}_{\mathrm{W}} \mathrm{I}+\mathrm{I}_{0}
$$

where, $\mathrm{k}_{\mathrm{W}} \mathrm{I}_{0}$ is the absolute threshold. The second part of Fechner's law is that the visual response R to intensity I is given by the Equation 16:

$$
\mathrm{R}=\log (\mathrm{I})
$$

The change in response is discrimination one. The discrimination threshold is denoted by $\Delta \mathrm{I}$. Then Equation 17:

$$
\Delta \mathrm{R}=\log \mathrm{I}+\Delta \mathrm{I}-\log \mathrm{I}
$$

Using the results for logarithms Equation 18 and 19:

$$
\begin{aligned}
& \Delta R=\log ^{\mathrm{I}+\Delta \mathrm{I}} \mathrm{I} \\
& \mathrm{e}^{\Delta \mathrm{R}}=\mathrm{e}^{\log ^{\mathrm{I}+\Delta \mathrm{I}} \mathrm{I}}
\end{aligned}
$$

Take the new constant $\mathrm{K}=\mathrm{e}^{\Delta \mathrm{R}}$ then Equation 20 to 24:

$$
\begin{aligned}
& \mathrm{K}=\mathrm{e}^{\log ^{\mathrm{I}+\Delta \mathrm{I}} \mathrm{I}} \\
& e^{\log K}=e^{\log I+\Delta I} \mathrm{I} \\
& \mathrm{K}={ }^{\mathrm{I}+\Delta \mathrm{I}} \mathrm{I} \\
& \Delta \mathrm{I}=\mathrm{I}(\mathrm{K}-1) \\
& \Delta \mathrm{I}=\mathrm{k}_{\mathrm{W}} \mathrm{I} \text { where } \mathrm{K}-1=\mathrm{k}_{\mathrm{W}}
\end{aligned}
$$

For some constant k. Norwich and Wong (1997) formulated the complete form of Fechner's law. They constituted the critical triplets for six cases. They concluded that rather than adhering to a rigid frame work of (1) standard law of sensation (2) functional form for the Weber fraction (3) constancy of the subjective JND, the unified view of psychophysics would follow this triplets.

Based on Lambertian reflectance model, the image can be expressed by Equation 25 and 26:

$\mathrm{Ix}, \mathrm{y}=\mathrm{L}(\mathrm{x}, \mathrm{y}) \times \mathrm{R}(\mathrm{x}, \mathrm{y})$

$\Delta \mathrm{I} \propto \Delta \mathrm{L}$

Jimenez-Sanchez et al. (2009) stated that the Weber's law can be expressed as Equation 27: 
$\mathrm{c}=\frac{\Delta \mathrm{L}}{\mathrm{L}}$

The above equation indicates that $\Delta(\log \mathrm{L})$ is proportional to c, so it can be expressed as Equation 28:

$$
\mathrm{c}-\mathrm{k} \log \mathrm{L}+\mathrm{b} ; \mathrm{L}>0
$$

where, $\mathrm{k}$ is constant and $\mathrm{b}$ being the background. In his approach, an approximation to Weber's law, the luminance $\mathrm{L}$ is proportional to the gray level intensity I of the image.

So Equation 29:

$\mathrm{c}-\mathrm{k} \log \mathrm{I}+\mathrm{b} ; \mathrm{L}>0$

This is the equation for MWLD. In this study, JND with MWLD is used to find the texture features. The algorithm for finding the background detection is given in section 4 .

\section{PROPOSED METHOD}

In this study, the fusion of color and texture features is used to identify the skin regions. This algorithm is implemented in two phases namely color feature extraction and texture feature extraction. The equation to find the skin feature of the image at $(\mathrm{x}, \mathrm{y})$ using the color feature and the texture feature is given by Equation 30:

$$
P(x, y)=T^{L} x, y+\beta * T^{\tau} x, y
$$

Where:

$\beta=$ The gain constant

$\mathrm{T}^{\mathrm{L}} \mathrm{x}, \mathrm{y}=$ The color feature

$\mathrm{T}^{\tau} \mathrm{x}, \mathrm{y}=$ The texture variations which is calculated by using MWLD and JND

\subsection{Color Feature Extraction}

In this hybrid approach, the results taken from Cheddad et al. (2009) and the $\mathrm{YC}_{\mathrm{b}} \mathrm{C}_{\mathrm{r}}$ skin color conditions based segmentation are combined to detect the skin tones. $\mu$ and $\sigma$ are calculated after fitting the data to normal distribution. If $\mu>0.02$, the empirical rule used to detect the skin tones is in Equation 9. If not, then $\mathrm{C}_{\mathrm{r}}$ is taken to find the skin tones. If the pixel intensity value of $\mathrm{C}_{\mathrm{r}}$ is greater than the gray threshold of $\mathrm{C}_{\mathrm{r}}$ then the pixel is considered as skin otherwise it is considered as nonskin. More number of skin regions is detected by using this approach. The explicitly defined region for $\mathrm{C}_{\mathrm{r}}$ components of $\mathrm{YC}_{\mathrm{b}} \mathrm{C}_{\mathrm{r}}$ is defined as Equation 31:

$$
\mathrm{f}_{\text {skin }} \mathrm{x}=\stackrel{\text { 1if } \mathrm{c}_{\mathrm{r}}>\operatorname{graythresh}\left(\mathrm{c}_{\mathrm{r}}\right)}{\text { 0elsewhere }}
$$

The skin image which is obtained by this hybrid approach is given in Fig. 4c. For comparision, the original and skin tone images are given in Fig. $4 \mathbf{a}$ and $\mathbf{b}$ respectively.

\subsection{Texture Feature Extraction}

Based on JND, the gradient variation at $(x, y)$ is given by Equation 32:

$\mathrm{g}_{\text {jnd }}^{\mathrm{i}} \mathrm{x}, \mathrm{y}=\mathrm{f}_{1} \mathrm{x}, \mathrm{y} * \mathrm{f}_{2} \mathrm{x}, \mathrm{y}$

where, $f_{1} x$, y represents the masking effect and $f_{2} x, y$ returns a value to represent the visibility threshold due to background luminance. According to Norwich and Wong (1997), the gradient variant is related to background of the image.

So Equation 33 and 34:

$\mathrm{g}_{\mathrm{k}} \mathrm{x}, \mathrm{y}=\log \mathrm{I}+\mathrm{b}_{\mathrm{g}} \mathrm{x}, \mathrm{y} \ldots . .$. Weber's Law

$$
\mathrm{T}^{\tau} \mathrm{x}, \mathrm{y}=\mathrm{k} * \log \mathrm{I}+\mathrm{g}_{\mathrm{b}} \mathrm{x}, \mathrm{y} * \mathrm{f}_{2} \mathrm{x}, \mathrm{y}
$$

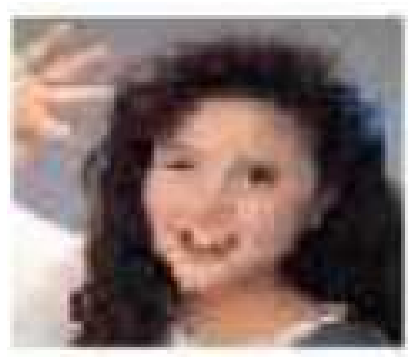

(a)

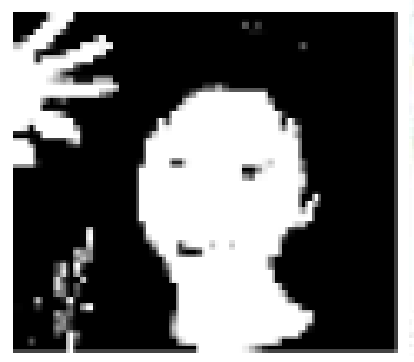

(b)

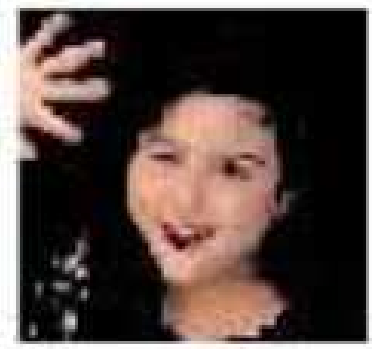

(c)

Fig. 4. Skin tone detection using hybrid approach (a) original image (b) skin tone image (c) skin image 
To find masking effect by using MWLD, $5 \times 5$ window is used. Here $\mathrm{I}$ is considered as the maximum weighted gradient $\mathrm{m}_{\mathrm{g}} \mathrm{x}, \mathrm{y} . \mathrm{m}_{\mathrm{g}} \mathrm{x}, \mathrm{y}$ is obtained by using the different operators $\mathrm{G} 1$ through $\mathrm{G} 4$ which is given below:

$$
\begin{array}{cccccccccc}
0 & 0 & 0 & 0 & 0 & 0 & 0 & 1 & 0 & 0 \\
1 & 3 & 8 & 3 & 1 & 1 & 3 & 8 & 0 & 0 \\
0 & 0 & 0 & 0 & 0 & 1 & 3 & 0 & -3 & -1 \\
-1 & -3 & -8 & -3 & 1 & 0 & 0 & -3 & -8 & 0 \\
0 & 0 & 0 & 0 & 0 & 0 & 0 & -1 & 0 & 0 \\
0 & 0 & 1 & 0 & 0 & 0 & 1 & 0 & -1 & 0 \\
0 & 0 & 3 & 8 & 0 & 0 & 3 & 0 & -3 & 0 \\
-1 & -3 & 0 & 3 & 1 & 0 & 8 & 0 & -8 & 0 \\
0 & -8 & -3 & 0 & 0 & 0 & 3 & 0 & -3 & 0 \\
0 & 0 & -1 & 0 & 0 & 0 & 1 & 0 & -1 & 0
\end{array}
$$

The steps to find the texture features of this proposed method is given below:

- $\mathrm{b}_{\mathrm{g}} \mathrm{x}, \mathrm{y}=\mathrm{a} \tan \left(\mathrm{c} * \frac{\mathrm{v} 00}{\mathrm{c} 01}\right)$ Where $\mathrm{c}$ is the ratio between the mean and standard deviation of the image.

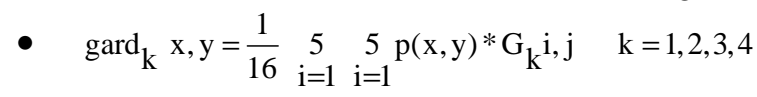

- $\mathrm{m}_{\mathrm{g}} \quad \mathrm{x}, \mathrm{y}=\max _{\mathrm{grad}} \mathrm{x}, \mathrm{y} \quad \mathrm{k}=1,2,3,4$

- $\mathrm{f}_{1} \mathrm{x}, \mathrm{y}=\mathrm{k} * \log \mathrm{m}_{\mathrm{g}} \mathrm{x}, \mathrm{y}+\mathrm{b}_{\mathrm{g}} \mathrm{x}, \mathrm{y}$

- $\mathrm{f}_{2} \mathrm{x}, \mathrm{y}=\mathrm{To} * 1-{\frac{\mathrm{b}_{\mathrm{g}} \mathrm{x}, \mathrm{y}}{127}}^{\frac{1}{2}}+3$ for $\mathrm{b}_{\mathrm{g}} \mathrm{x}, \mathrm{y} \leq 127$

$$
\gamma^{*} b_{g} x, y-1+3 \text { for } b_{g} x, y>127
$$

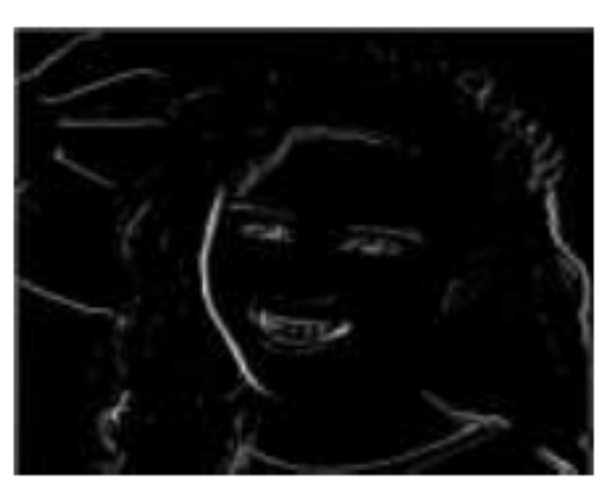

(a)
The output image of ' $f 1$ ' in texture feature extraction and the resulting texture images are depicted in Fig. 5a and $\mathbf{b}$ respectively. It is desirable to obtain a function that resembles image background without using morphological erosion and dilation, since these morphological transformations generate new contours when structuring element is increased. There is another class of transformation that allows the filtering of the image without generating the new components. This transformation is called transformation by reconstruction. The algorithm to find $b_{g} x, y$ starts by calculating the average gray level through a $5 \times 5$ window around the current sample with the coordinates $\mathrm{x}$ and $\mathrm{y}$. To in $\mathrm{f}_{2}$ marks the visibility threshold when the background gray level is zero and $\gamma$ is the slope of the line that models the function at higher background luminance.

The images which are obtained by color and texture features are fused together to get the skin pixels. The hypothesis of this study is the inclusion of texture features that increases the separability of skin and nonskin pixels. To provide the evidence for this hypothesis, the proposed algorithm was tested on images with different background and illumination conditions. The proposed method is also compared with several states of the art. Wang et al. (2011) used this Weber's Law for face recognition. To obtain the Weber's Face, the term is designed for adjusting the intensity difference between the neighboring pixels. But in this proposed method, no such constant term is used for intensity adjustment. For every image, the ratio between the mean to standard deviation of the given image is used as c. For calculating the JND of the image, the constant term $\mathrm{k}$ is used. Figure 6 shows the relation between $k$ and the accuracy. As can be seen, $\mathrm{k}=0.3$ yields better accuracy.

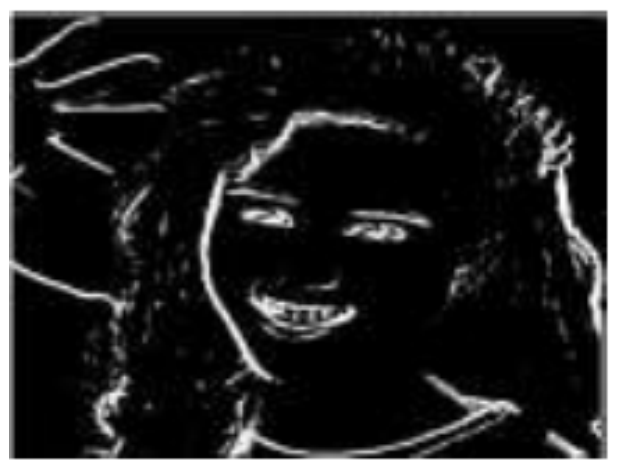

(b)

Fig. 5. (a) Output image of f1 (b) Texture image 


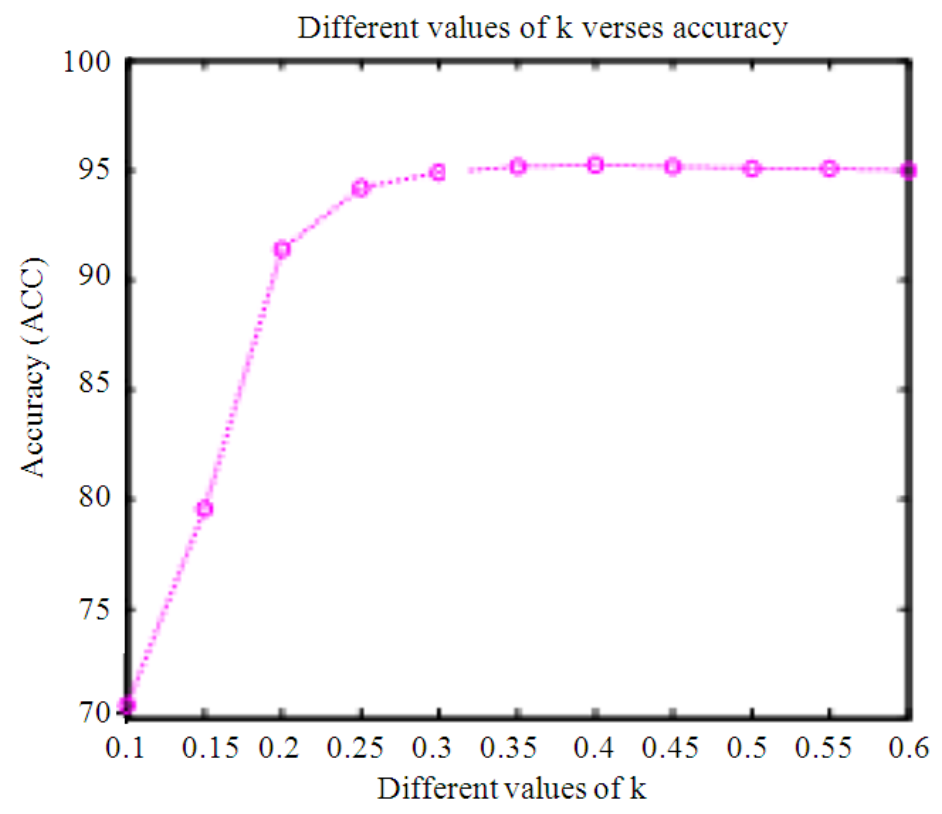

Fig. 6. Graph to find the optimum value for $\mathrm{k}$

From Fig. 6, if $\mathrm{k}>0.3$ the accuracy of this method is constant. The optimized value of $\mathrm{k}$ is taken as 0.3 .

\section{EXPERIMENTAL RESUULTS}

An extensive experimental study was carried out to investigate the effectiveness of the proposed method. For getting experimental results, Markess Weber' data set of Californine. Ins. of tech containing 450 pieces of 27 individual persons in different brightness, posture and background are used and 10 images are taken from internet. Generally the performance of the detection method is assessed by Receiver Operating Analysis (ROC) and Area Under Curve (AUC). ROC graph is a technique for visualizing, organizing and selecting the methods based on their performance. True Positive, True Negative, False Positive and False Negative are the parameters used to depict the characteristic of ROC. The Fig. 7 shows the overall idea of ROC.

The evaluation of detection methods and comparative analysis of the proposed method are mainly based on the two parameters True Positive Rate (TPR) and False Positive Rate (FPR). These two parameters are taken to draw the ROC curve. TPR and FPR are given by:

$\mathrm{TPR}=$ Total no. of pixels detected correctly as Skin Pixels/Total No. of Skin Pixels= TP/P
FPR $=$ Total no. of pixels detected correctly as Non Skin Pixels/Total No. of Non Skin Pixels = FP/N

Area under ROC is used to analyze the performance of the proposed method. If two models have same FPR, high TPR is considered as good one. The reverse process is taken for high FPR also. The ROC curve for the proposed and the existing methods are plotted in Fig. 8 which is possible to analyze and compare the proposed method with the existing methods. AUC for the proposed method is high compared with the existing methods. It shows that for medium to high TPR, the proposed method performs well. Reversely, for low TPR, all methods are performed similarly.

Depicted in Fig. 9 is the comparison between original image and the skin tone images of the existing methods of $\mathrm{YC}_{\mathrm{b}} \mathrm{C}_{\mathrm{r}}$, (Cheddad et al., 2009; Prema and Manimegalai, 2012) and the proposed method. The images given in Fig. 9 are the sample images taken from internet and the image in the above said data set. Figure 9 shows that the proposed algorithm is insensitive to false alarms.

Figure 9 Skin Detection (Left to Right) Original Input Image (toy.jpg in table), skin tone detection by $\mathrm{YC}_{\mathrm{b}} \mathrm{C}_{\mathrm{r}}$, (Cheddad et al., 2009; Prema and Manimegalai, 2012) and the proposed method. 
In this method, for getting texture features, windows with different sizes such as $3 \times 3,5 \times 5,7 \times 7$ are used. TPR for different windows of this proposed method is shown in Fig. 10. Linear variations in TPR were obtained for $5 \mathrm{X} 5$ window. For other window sizes, variations are not smooth one. So, $5 \times 5$ window is used in the proposed method to obtain the skin tones.

Manually selected ground truths, TPR and FPR are obtained for the above data set. Figure 10 shows the performance analysis of this proposed method against the existing explicitly defined method such as RGB, $\mathrm{YC}_{\mathrm{b}} \mathrm{C}_{\mathrm{r}}$, (Cheddad et al., 2009; Prema and Manimegalai, 2012). As can be seen, the proposed method preserves lower rate for false positives while securing a high for true positives among all methods. It is evident that TPR of this method follows a straight line compared to others. That is the proposed method does not depend on the background conditions of the image. The proposed method gives nearly constant value for TPR verses FPR of the images in the dataset taken for analysis.

In Fig. 11, TPR for different images are plotted for the proposed and existing methods. In the proposed method, nearly linear graph is obtained. It proves the relative quality of the proposed method. By the inclusion of texture with color spaces, FPR of the given image is reduced. In some cases both the existing and the proposed methods produce the same FPR value which gives the alarm of improper selection of the image. In Table 1 and 2, the TPRs and FPRs for different window sizes and the methods are given. Table 1 reveals that the illumination compensation and color transformation can improve the performance of the detection rate for some images. But the great impact in detection rate is achieved by the algorithm used. Detection rate of the proposed method is high as shown in Table 2 .

Table 1. Comparison of the proposed method for various window sizes

\begin{tabular}{lcccccc}
\hline & $3 \times 3$ Window & & $5 \times 5$ Window & & \multicolumn{2}{c}{$7 \times 7$ Window } \\
Image No. & TPR & FPR & TPR & FPR & TPR & FPR \\
\hline Toy & 96.52 & 1.26 & 97.73 & 0.66 & 97.48 & 0.52 \\
test2 & 100.00 & 0.00 & 99.92 & 0.03 & 90.91 & 0.03 \\
nadal & 88.16 & 2.39 & 89.56 & 1.85 & 91.72 & 0.98 \\
1 & 94.46 & 0.70 & 95.76 & 0.29 & 95.16 & 0.15 \\
180 & 84.16 & 2.71 & 92.90 & 0.75 & 91.31 & 0.48 \\
90 & 85.05 & 1.31 & 89.99 & 0.44 & 85.82 & 0.28 \\
47 & 98.84 & 0.14 & 93.03 & 0.08 & 99.36 & 0.03 \\
60 & 86.61 & 2.31 & 84.96 & 1.79 & 84.90 & 1.07 \\
22 & 88.00 & 1.81 & 87.74 & 4.14 & 87.98 & 0.67 \\
195 & 71.90 & 3.63 & 69.57 & 8.71 & 40.28 & 0.60 \\
196 & 55.89 & 7.61 & 56.88 & 0.07 & 100.00 & 4.55 \\
217 & 94.48 & 0.04 & 99.36 & 3.44 & 94.70 & 0.00 \\
242 & 84.17 & 2.14 & 79.36 & 14.70 & 51.58 & 0.24 \\
264 & 57.48 & 10.98 & 54.28 & 2.72 & 95.42 & 4.63 \\
269 & 88.49 & 1.95 & 85.78 & 5.82 & 73.70 & 0.32 \\
288 & 76.86 & 4.41 & 73.32 & 1.56 & 91.45 & 1.95 \\
308 & 95.80 & 0.82 & 93.06 & 2.16 & 95.14 & 0.31 \\
315 & 90.99 & 1.51 & 89.66 & & & 0.26 \\
\hline
\end{tabular}

Table 2. Comparison between the proposed and existing methods

\begin{tabular}{|c|c|c|c|c|c|c|c|c|c|c|}
\hline \multirow{2}{*}{$\begin{array}{l}\text { Methods/ } \\
\text { image name } \\
\text { and no }\end{array}$} & \multicolumn{2}{|l|}{ RGB } & \multicolumn{2}{|c|}{$\mathrm{YCbCr}$} & \multicolumn{2}{|c|}{$\begin{array}{l}\text { Algorithm used in } \\
\text { Cheddad } \text { et al. (2009) }\end{array}$} & \multicolumn{2}{|c|}{$\begin{array}{l}\text { Algorithm used } \\
\text { in (Prema and } \\
\text { Manimegalai (2012) }\end{array}$} & \multicolumn{2}{|c|}{ Proposed } \\
\hline & TPR & FPR & TPR & FPR & TPR & FPR & TPR & FPR & TPR & FPR \\
\hline Toy & 89.84 & 7.16 & 69.51 & 16.37 & 89.84 & 4.52 & 69.51 & 21.79 & 93.35 & 2.61 \\
\hline 1 & 78.75 & 1.14 & 62.89 & 1.02 & 82.89 & 4.14 & 78.75 & 5.62 & 92.15 & 1.20 \\
\hline Nadal & 78.23 & 5.37 & 80.32 & 10.36 & 80.32 & 5.37 & 70.23 & 8.68 & 86.24 & 3.11 \\
\hline
\end{tabular}


Prema, C. and D. Manimegalai / Journal of Computer Science 10 (9): 1680-1691, 2014

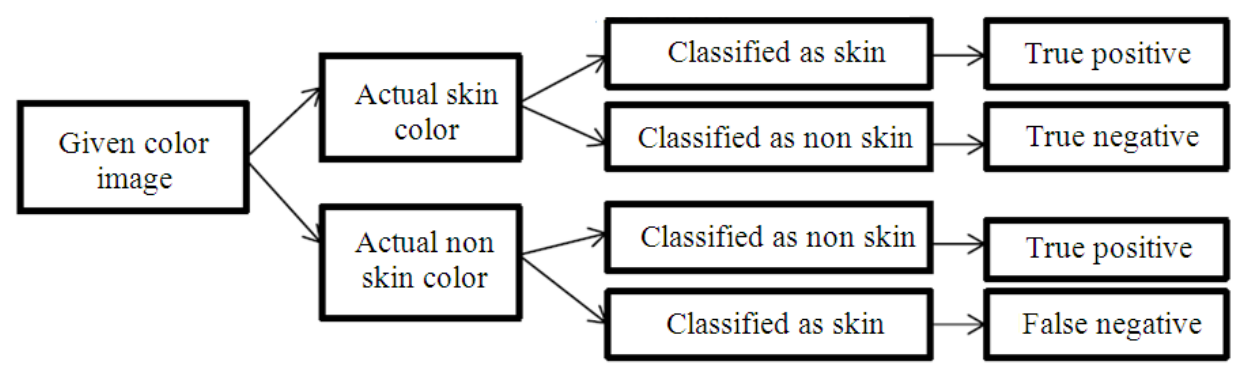

Fig. 7. General Description of ROC

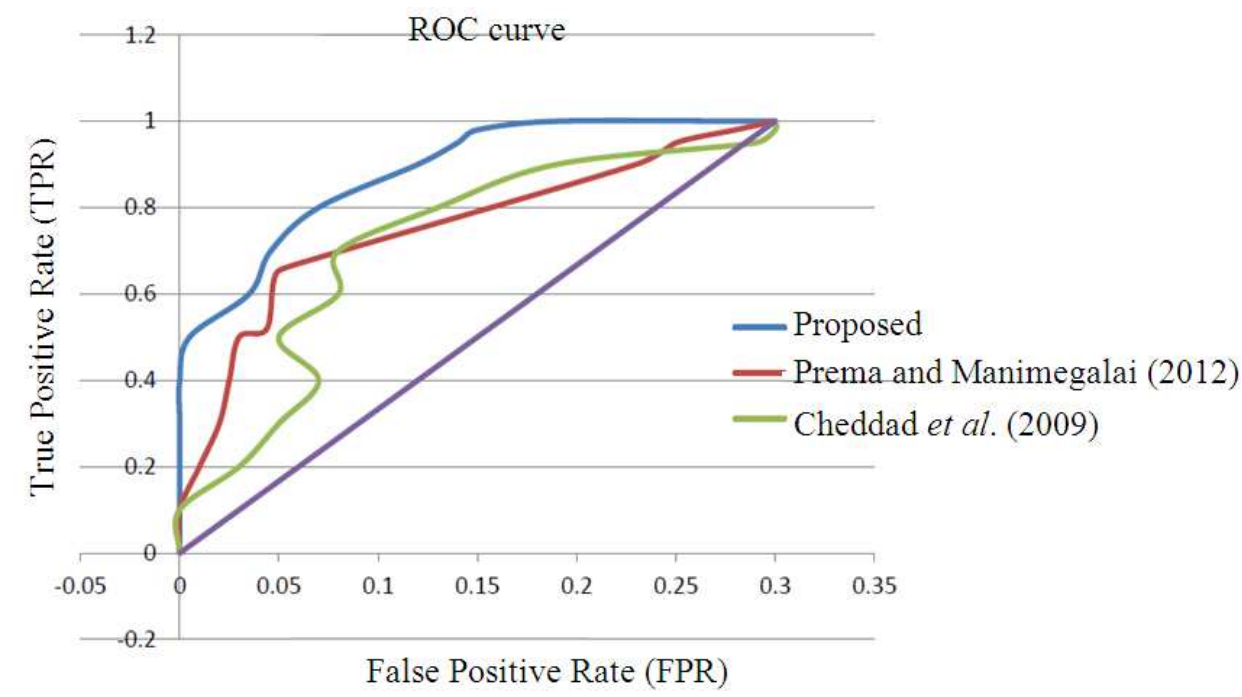

Fig. 8. ROC curve for the proposed method with (Prema and Manimegalai, 2012) and (Cheddad et al., 2009)
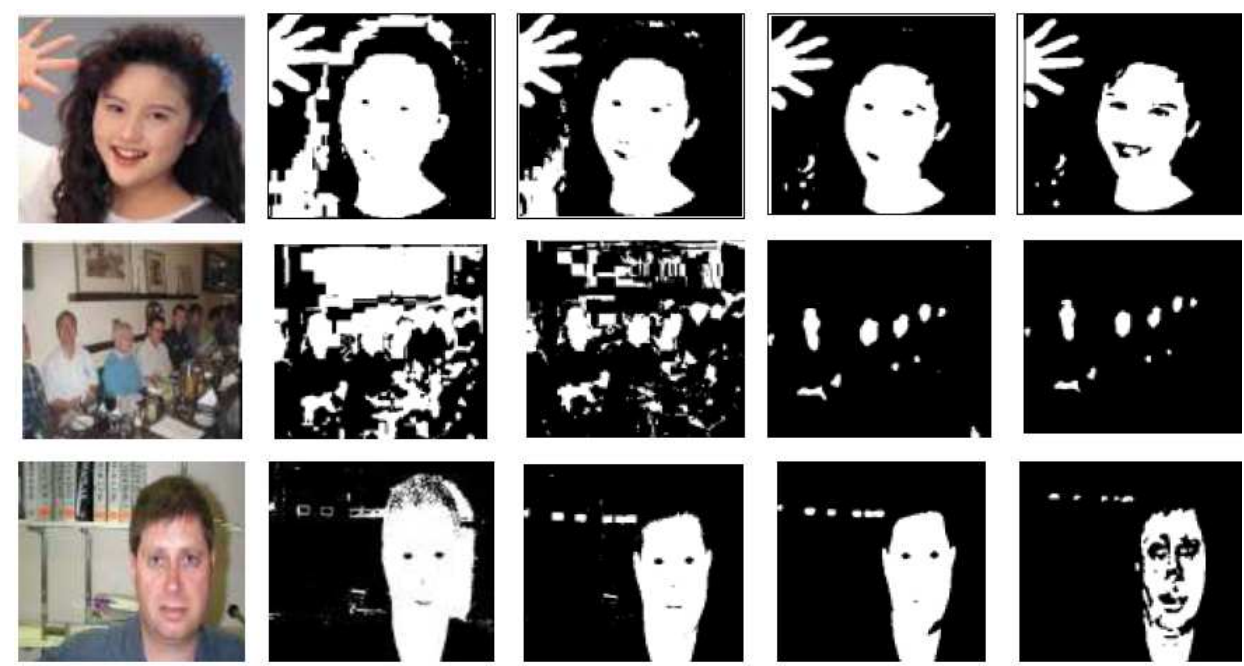

Fig. 9. Skin Detection (Left to Right) Original Input Image (toy.jpg in table), skin tone detection by $\mathrm{YC}_{\mathrm{b}} \mathrm{C}_{\mathrm{r}}$, $($ Cheddad et al., 2009), (Prema and Manimegalai, 2012) and the proposed method 
Prema, C. and D. Manimegalai / Journal of Computer Science 10 (9): 1680-1691, 2014

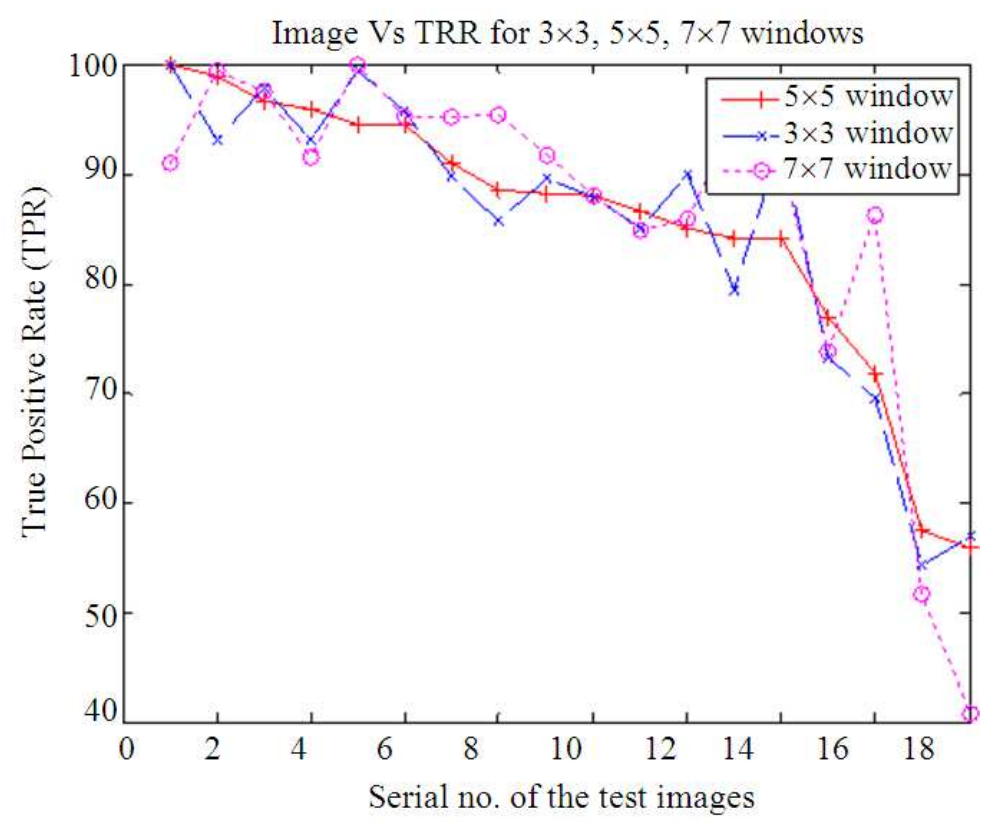

Fig. 10. Performance evaluation of the proposed method with different windows

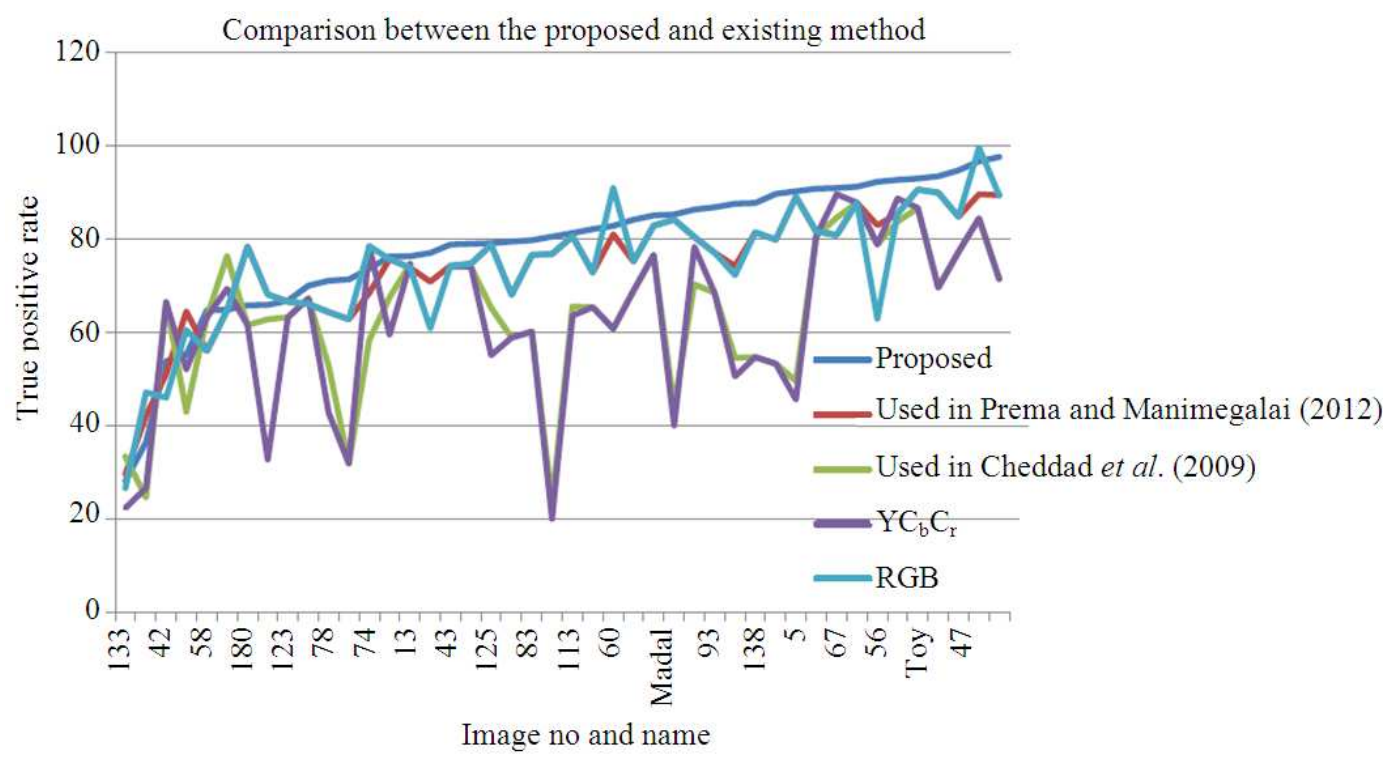

Fig. 11. Performance evaluation of the proposed and existing methods

\section{CONCLUSION}

In this study, a simple and efficient method for human skin detection is proposed. By the use of JND, no illumination compensation is needed. In this proposed method, both color and texture features of the images are combined to obtain the skin tones. By the use of textures, more skin tones are detected by comparing the existing methods. Experimental results show that the proposed method achieved satisfactory performance; it can reduce FPR and increase TPR at the same time. Necessary future work is to validate 
the proposed algorithm using a standard skin database like the ECU data set (Phung et al., 2005) and Compaq data set (Jones and Rehg, 1999).

\section{REFERENCES}

Hsu, R.L., M. Abdel-Mottaleb and A.K. Jain, 2002. Face detection in color images. IEEE Trans. Patt. Anal. Mach. Intell., 24: 696-706. DOI: 10.1109/34.1000242

Shin, M.C., K.I. Chang and L.V. Tsap, 2002. Does color space transformation make any difference on skin detection? Proceedings of the IEEE Workshop on Applications of Computer Vision, (WACV '02), pp: 275-279.

Prema, C. and D. Manimegalai, 2012. A novel skin tone detection using hybrid approach by new color space. Int. J. Comput. Applic., 46: 15-19. DOI: 10.5120/6919-9259

Liu, C. and H. Wechsler, 2002. Gabor feature based classification using the enhanced fisher linear discriminant model for face recognition. IEEE Trans. Image Process, 11: 467-476. DOI: 10.1109/TIP.2002.999679

Ojala, T., M. Pietikainen and T. Maenpaa, 2002. Multiresolution gray-scale and rotation invariant texture classification with local binary patterns. IEEE Trans. Patt. Anal. Mach. Intell., 24: 971-987. DOI: 10.1109/TPAMI.2002.1017623

Ahonen, T., A. Hadid and M. Pietikanien, 2006. Face description with local binary pattern: Application to face recognition. IEEE Trans. Patt. Anal. Mach. Intell., 28: 2037-2041. DOI: 10.1109/TPAMI.2006.244

Caulsi, D.A. and M.E. Jernigam, 2000. Designing gabor filters for optimal texture separability. Patt. Recogn. 33: $1835-1849 . \quad$ DOI: $10.1016 /$ S00313203(99)00181-8

Chen, J., S. Shan, C. He, G. Zhao and M. Pietikainen et al. 2010. WLD: A robust local image descriptor. IEEE Trans. Patt. Anal. Mach. Intell., 32: 1705-1720. DOI: 10.1109/TPAMI.2009.155

Gong, D., S. Li and Y. Xiang, 2011. Face recognition using the Weber local descriptor. Proceedings of the Asian Conference on Pattern Recognition, Nov. 2828, IEEE Xplore Press, Beijing, pp: 589-592. DOI: 10.1109/ACPR.2011.6166675

Wang, B., W. Li, W. Yang and O. Liao, 2011. Illumination normalization based on Weber's law with application to face recognition. IEEE Trans. Sgnal Proc., 18: 462-465. DOI: 10.1109/LSP.2011.2158998
Maenpaa, T. and M. Pietikäinen, 2004. classification with color and texture: Jointly or separately? Patt. Recogn., 37: 1629-1640.

Taqa, A.Y. and H.A. Jalab, 2010. Increasing the reliability of fuzzy inference system-based skin detector. Am. J. Applied Sci., 7: 1129-1138. DOI: 10.3844/ajassp.2010.1129.1138

Mustafa, R. and D. Zhu, 2013. Objectionable image detection in cloud computing paradigm-a review. J. Comput. Sci., 9: 1715-1721, 2013. DOI: 10.3844/jcssp.2013.1715.1721

Norwich, K.H. and W. Wong, 1997. Unification Psycophysical Phenomena: The complete form of fechner's law. Percept. Psycophys., 59: 929-940. PMID: 9270366

Jimenez-Sanchez, A.R., J.D. Mendiola-Santibanez, I.R. Terol-Villalobos, G. Herrera-Ruiz and D. VargasVazquez et al., 2009. Morphological background detection and enhancement of images with poor lighting. IEEE Image Process., 18: 613-623. DOI: 10.1109/TIP.2008.2010152

Phung, S.L., A. Bouzerdoum and D. Chai, 2005. Skin segmentation using color pixel classification: analysis and comparison. IEEE Trans. Patt. Anal. Mach. Intell., 27: 148-154. DOI: 10.1109/TPAMI.2005.17

Jones, M.J. and J.M. Rehg, 1999. Statistical color models with application to skin detection. Proceedings of the IEEE Computer Society Conference on Computer Vision and Pattern Recognition, Jun. 2325, IEEE Xplore Press, Fort Collins, DOI: 10.1109/CVPR.1999.786951

Vezhnevets, V., V. Sazonov and A. Andreeva, 2003. A survey on pixel based skin-color detection techniques. Moscow State University.

Cheddad, A., J. Condell, K. Curran and P. McKevitt, 2009. A new colour space for skin tone detection. Proceedings of the 16th IEEE International Conference on Image Processing, Nov. 7-10, IEEE Xplore Press, Cairo, pp: 497-500. DOI: 10.1109/ICIP.2009.5413947 